\title{
Analysis of solitary wave impulses in granular chains using ultrasonic excitation
}

\author{
J. Yang, ${ }^{1, *}$ D. A. Hutchins, ${ }^{1}$ O. Akanji, ${ }^{1}$ P. J. Thomas, ${ }^{1}$ L. A. J. Davis, ${ }^{1}$ S. Harput,${ }^{2}$ P. Gelat,${ }^{3}$ S. Freear, ${ }^{2}$ and N. Saffari ${ }^{3}$ \\ ${ }^{1}$ School of Engineering, University of Warwick, Coventry CV4 7AL, United Kingdom \\ ${ }^{2}$ School of Electronic and Electrical Engineering, University of Leeds, Leeds LS2 9JT, United Kingdom \\ ${ }^{3}$ Department of Mechanical Engineering,University College London, Torrington Place, London WC1E 7JE, United Kingdom
}

(Received 15 August 2015; revised manuscript received 3 January 2016; published 10 June 2016)

\begin{abstract}
The propagation of broad bandwidth solitary wave impulses, generated within granular chains by narrow bandwidth ultrasonic excitation, is studied in detail. Theoretical predictions are compared to experimental results. It is demonstrated that the observed effects result from a sum of a solitary wave traveling out from the source with a wave that reflects from the far end of the chain. It is shown that this combination, when used with an excitation in the form of a long-duration tone burst, encourages the generation of multiple impulses with a characteristic periodicity. This study shows that the properties of the chain structure and the excitation can be adjusted so as to generate ultrasonic solitary wave impulses with a high amplitude and known frequency content, which are of interest in applications such as biomedical ultrasound.
\end{abstract}

DOI: 10.1103/PhysRevE.93.063002

\section{INTRODUCTION}

One-dimensional (1D) granular chains are known to support solitary waves due to nonlinear Hertzian contact between neighboring particles [1-4]. The properties of these solitary waves have been studied in some detail [5-9], and it is known that wave transmission in a particular chain depends on the level of the static precompression force relative to the applied transient input signal, and the resulting behavior can be classified as being linear, weakly nonlinear, or highly nonlinear $[10,11]$. Solitary waves have been observed experimentally using impulsive input signals (e.g., $[2,4,5]$ ). The solitary wave speed is much smaller than the bulk sound speed within the material making up the granular medium (often spheres in these studies), but can be varied by changing the magnitude of the applied static precompression force. This led to the concept of varying the phase of signals along a chain of spheres, leading to a tunable focus and the very interesting concept of sound bullets [11-13].

Weakly compressed granular chains can generate solitary waves as a highly nonlinear mode, leading to the concept of a "sonic vacuum" due to the lack of transmission of classical linear waves through them [4]. In the continuum approximation limit, the existence of a family of solitary waves can be predicted in an analytical solution using a long-wave approximation [1,4] in an infinite granular chain, and the nature of solitary waves in such chains has been analyzed in some detail [14-16]. However, the situation is different if finite chain lengths are considered [17,18]. Jayaprakash et al. [18] studied such chains with fixed boundary conditions, and indicated the presence of an in-phase nonlinear normal mode (NNM) which resembles a traveling wave propagating backward and forward through the finite granular chain. Note

\footnotetext{
*jia.yang@warwick.ac.uk
}

Published by the American Physical Society under the terms of the Creative Commons Attribution 3.0 License. Further distribution of this work must maintain attribution to the author(s) and the published article's title, journal citation, and DOI. that NNMs are usually defined as synchronous period solutions of the nonlinear equations of motion of dynamical systems; however, Jayaprakash et al. [18] extended the definition to include time-periodic oscillations where each granule or sphere oscillates with an identical frequency, but they are not necessarily synchronous with each other. Under these conditions, the in-phase NNM can represent a traveling wave in a granular chain. This in-phase NNM also allows the particles to temporarily lose contact, a situation that is predicted to exist in the present system. Jayaprakash et al. [18] also predicted the presence of frequency bands which were denoted as propagation and attenuation bands (PBs and $\mathrm{ABs}$ ) for an infinite number of identical spherical beads.

These frequency bands have been observed in a two-bead experimental system [19]. The above research results are important because they exhibit the existence of periodic traveling waves under the situation of a sonic vacuum. Thus the generation of high frequency solitonlike impulses becomes possible. In current biomedical applications, there are fundamental limits on the spatial resolution and power densities that can be achieved using linear transduction methods, so the authors were motivated to discover solitary wave impulses in experiments due to the potential of important biomedical applications.

In the majority of the experiments that have been reported, solitary waves were generated using mechanical impact by a striker, although this excitation method is not particularly convenient for practical use, as the excitation tends to be of low frequency. In order to generate solitary wave impulses within granular media at high enough frequencies, a new excitation method is needed. In recent years, new excitation methods using ultrasonic transducers and piezoelectric actuators have been investigated for use with granular media. For example, transient signals from piezoelectric actuators have been used to generate impulses within chains of spheres of $\sim 9 \mathrm{~mm}$ diameter, and interesting effects observed [13].

The authors started to pursue the solitary wave impulses using ultrasonic excitations. Although the cutoff frequency $\left(f_{c}\right)$ of a 1D granular chain is derived based on a linear assumption [2], the relation between $f_{c}$ and the size and material of the spheres in the chain, assumed to be under a very 
small static force, is still considered as an important design consideration. Another limitation while choosing an appropriate excitation transducer is the trade-off between frequency and output displacement. High frequency transducers tend to produce low output displacements, which decreases further with damping within the transducer. Taking these factors into account, we have constructed an experimental system using a $73-\mathrm{kHz}$ ultrasonic horn, this being a resonant device with a high amplitude output. The higher excitation frequency then led to the use of smaller steel spheres of $1 \mathrm{~mm}$ diameter, with the aim of creating higher frequency solitary wave impulses.

Our initial studies [20] have indicated that ultrasonic excitation of finite-length chains can lead to the generation of impulses with broad bandwidths. This paper studies the fundamental dynamics of these types of ultrasonic signals, and explores the mechanisms whereby the solitary wave impulses are generated.

Section II introduces the experimental setup and conditions needed to create the solitary wave impulses. The resultant impulses and frequency bands for different length chains are presented and analyzed. In Sec. III, the discrete dynamic equations of spheres in a chain based on Hertzian contact are given, and a numerical algorithm is developed to simulate the experimental system. The nonsmooth dynamic behavior in granular media is exhibited and the NNMs are studied by solving the discrete equations of motion numerically. Good agreement between theoretical predictions and experimental results is obtained. The motions of individual particles within the chain are also examined theoretically, where it is shown that the characteristics of the in-phase NNM are exhibited in the traveling waves. In Sec. IV the formation of solitary waves with characteristic lengths is also identified by comparison of the numerical simulations to existing theoretical approaches $[1,4]$. This theoretical analysis reveals the effect of both the chain characteristics and the input signal on the creation of periodic solitary wave impulses in finite-length granular media.

\section{EXPERIMENTAL GENERATION OF SOLITARY WAVE IMPULSES}

The experimental arrangement for a single chain of 1-mmdiameter chrome steel spheres is shown schematically in Fig. 1. Cylindrical holders for different length chains were fabricated from photopolymer resin using microstereolithography, a form of additive manufacturing. The spheres were placed in the holder and initially touching, with the static precompression near zero. The first sphere was excited harmonically by a longitudinal ultrasonic horn, which was driven by an Agilent
33120A Function/Arbitrary waveform generator and a power amplifier. This produced a resonant response with a high amplitude at $73 \mathrm{kHz}$. The last sphere was positioned against an aperture, so that the particle velocity waveform could be measured using a Polytec laser vibrometry system. The velocity signal of both the tip of the ultrasonic horn and that of the last sphere in the chain were both recorded in this way, and are referred to, respectively, in the following as the input and output signals of the granular chain. The voltage drive waveform into the horn was in the form of a tone burst at $73 \mathrm{kHz}$, of adjustable duration, although the horn tip motion was an elongated version of this because of its own resonant response.

For a fixed length chain, the drive amplitude could be gradually increased in order to observe the change of the output pulses and obtain the threshold value of generation of solitary wave impulses. It was found that the best results were obtained for a specific number of cycles for different chain lengths. Some example waveforms are shown in Fig. 2 for a chain of six spheres, with the corresponding frequency spectra presented in Fig. 3. The amplitude in the amplitude spectra plots is the vector magnitude of the two Fourier series coefficients for each frequency, which are derived from a fast-Fourier transform of the velocity signals. A fixed tone-burst length of 20 cycles was used. At low input amplitudes, weakly nonlinear signals were observed and fewer harmonics were excited, as shown in Figs. 2(a) and 3(a). As the voltage increased [Figs. 2(b) and 3(b)], more nonlinear features started to appear, in this case two subharmonics and some higher order harmonics. However, when the input voltage increased further, strongly nonlinear signals were present as ultrasonic impulses at high amplitude were generated [Figs. 2(c) and 3(c)]. The resultant waveform exhibits broad bandwidth ultrasonic signals, with strong high order harmonics and subharmonics of the original forcing frequency being present in the spectrum. These solitary wave impulses exhibit the feature of a characteristic periodicity, with the output signals eventually damped due to dissipation and energy losses into the polymer holder after the excitation is stopped. The results indicate that solitary wave impulses can be created once the drive amplitude attains a specified threshold value for a given excitation input waveform.

A train of impulses can also be observed in other chain lengths, as shown in Fig. 4 for chains of three and ten spheres. However, we have not observed the solitary wave impulses in other length chains within the three to ten sphere range using similar input conditions, indicating that the chain length has to match the particular input frequency for solitary wave impulses to exist. There are some important features observed in the solitary wave impulses observed in the three different chain

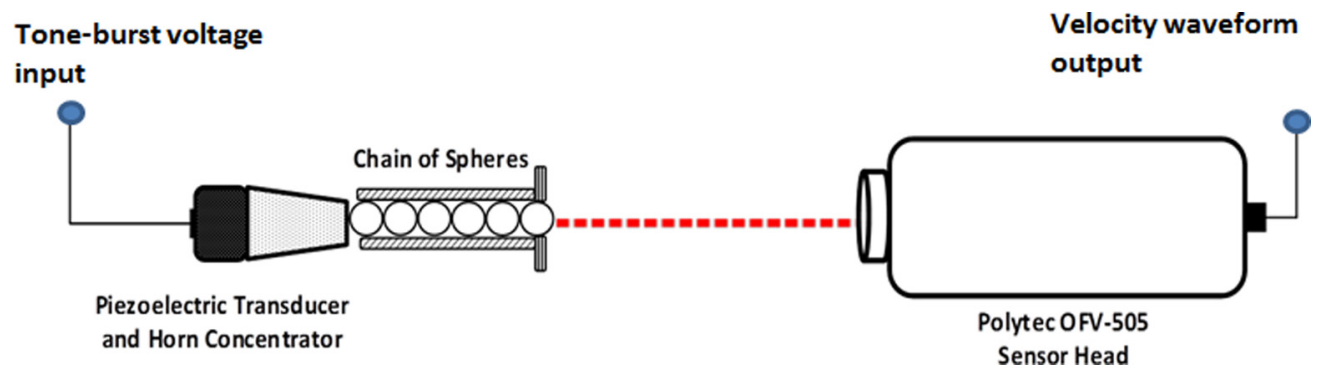

FIG. 1. Experimental arrangement. 


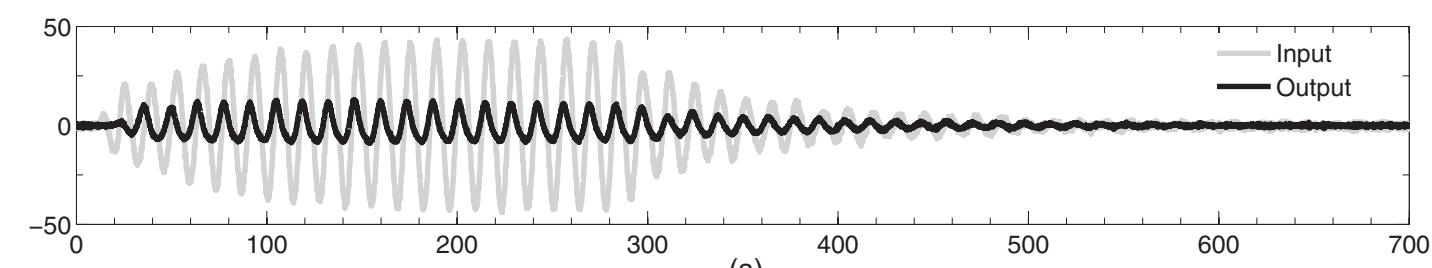

(a)

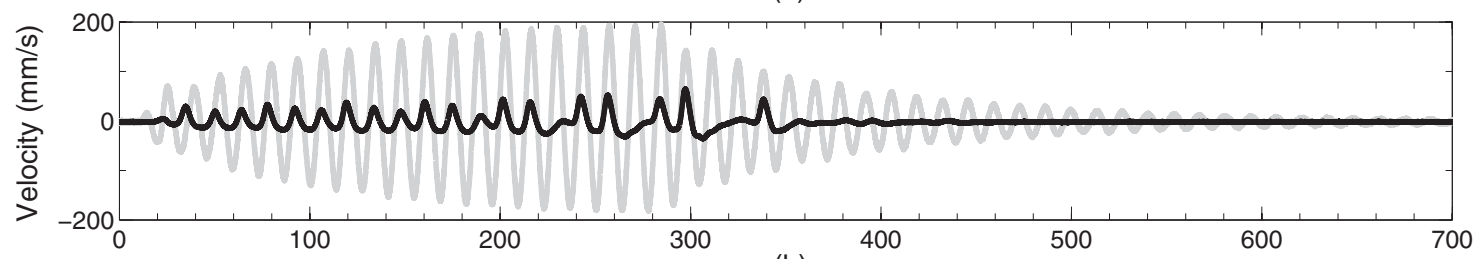

(b)

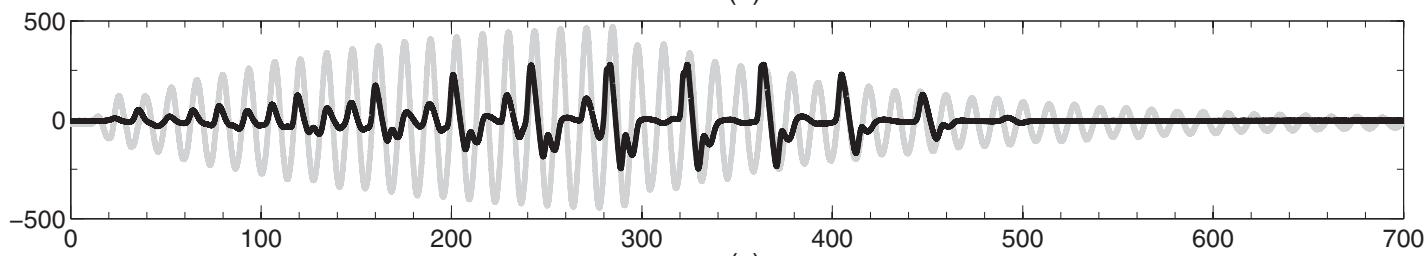

(c)

Time $(\mu s)$

FIG. 2. The input (light trace) and output (dark trace) signals in a chain of six spheres as the input amplitude from the horn was gradually increased.

lengths. As shown in Figs. 3(c) and 4, the frequency of the lowest subharmonic for chains of three, six, and ten spheres is $1 / 2,1 / 3$, and $1 / 4$ that of the input frequency, respectively. This indicates that the spectrum makes a transition toward lower frequencies as the number of particles increases. The longer chain has more closely spaced harmonics, which also extends to higher frequencies. In addition, the period of the impulses in each chain is found to be the reciprocal of the lowest harmonic in each spectrum, i.e., 27, 41, and $55 \mu$ s for chains of three, six, and ten spheres, respectively. These findings exhibit different characteristics to those seen in traditional solitary wave propagation in chains of spheres, in that a high amplitude sinusoidal wave has generated a set of periodic impulses. The rest of this paper explores the natures of these ultrasonic signals, and compares their properties to those expected in infinite chains. Conclusions can then be drawn concerning the existence of solitary waves and the role of reflections within the chain.

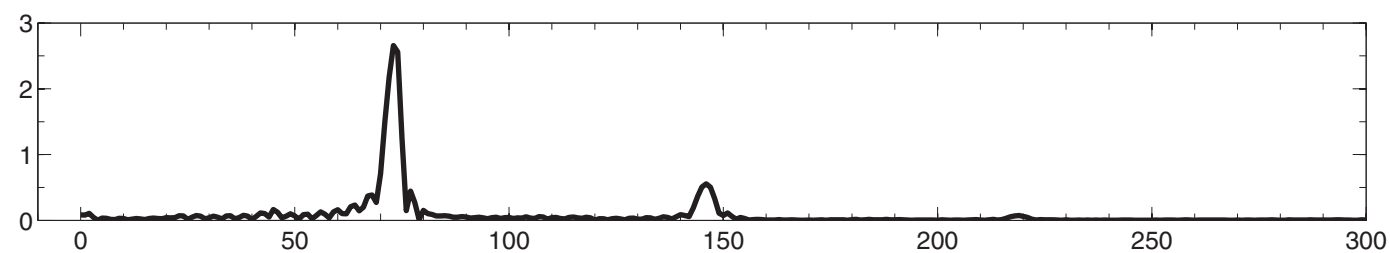

(a)

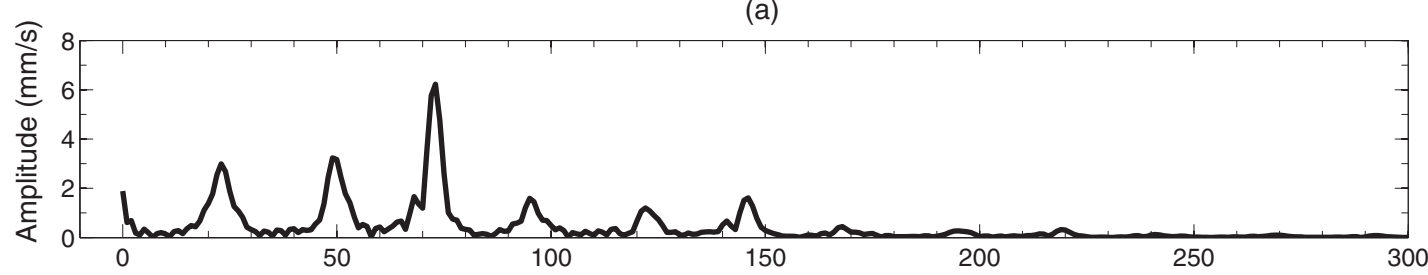

(b)

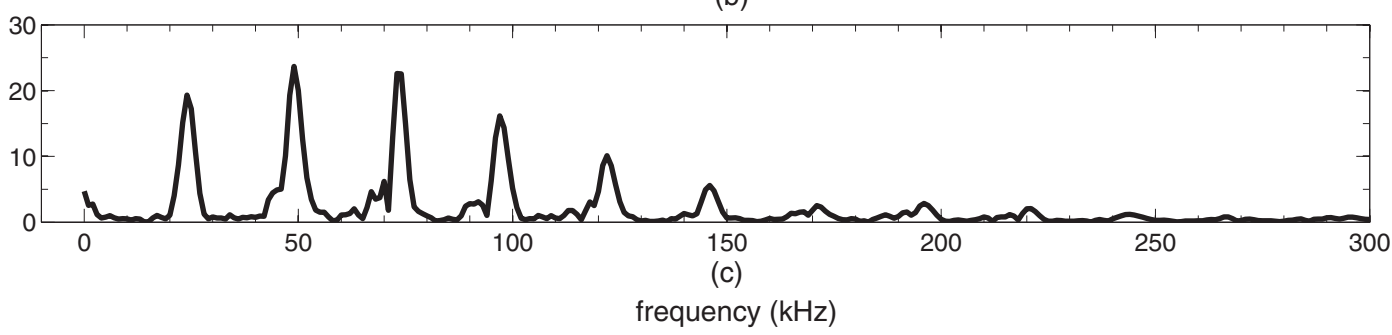

FIG. 3. Frequency spectra of the output signals shown in Fig. 2. 

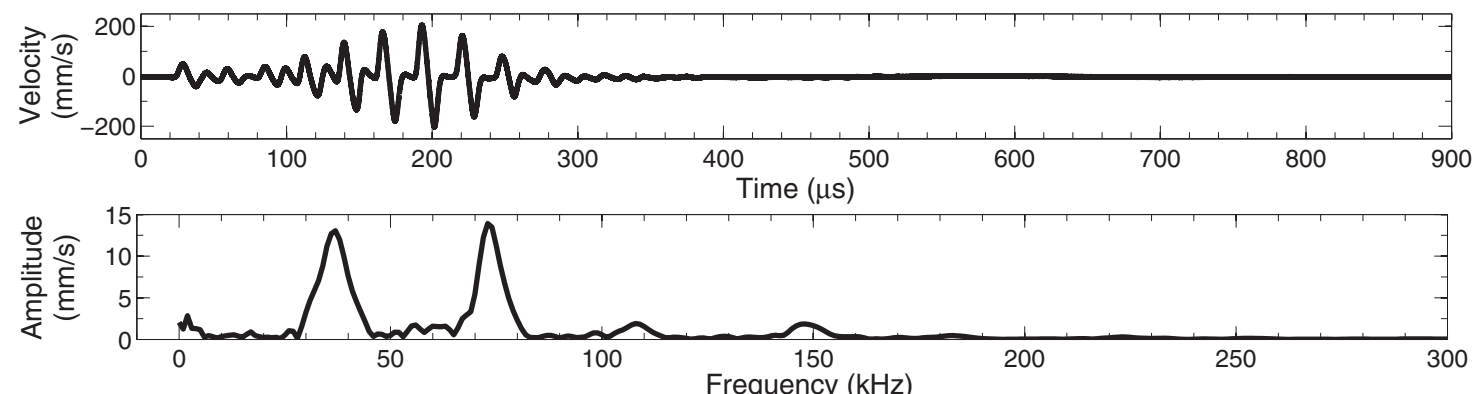

(a)
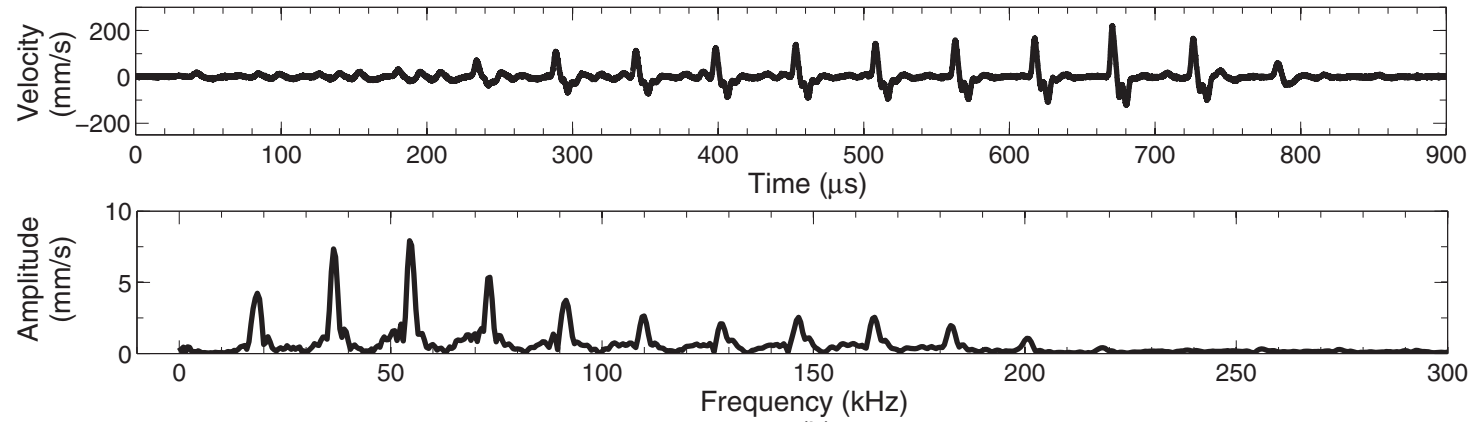

(b)

FIG. 4. Waveforms and spectra measured by experiments for the different length chains. (a) Three-sphere chain; (b) ten-sphere chain.

\section{THEORETICAL PREDICTIONS OF EXPERIMENTAL BEHAVIOR}

\section{A. Discrete dynamic modeling of chains of spheres}

The equations of motion for a finite-length spherical chain were presented in an earlier paper [20], but are included here for clarity. Deformation between particles was assumed to be within the elastic range, so that the interaction between identical spherical particles can be described by the nonlinear Hertzian law [4]:

$$
F=\frac{\sqrt{2 R} E}{3\left(1-v^{2}\right)}\left[2 R-\left(x_{2}-x_{1}\right)\right]^{3 / 2},
$$

where $F$ is the force between adjacent spheres, $E$ is the Young's modulus of the material with a Poisson's ration $v$, and $x_{1}$ and $x_{2}$ are the center coordinates of the spheres $\left(x_{2}>x_{1}\right)$. The spheres have an identical mass $(m)$ and radius $(R)$, with the first sphere in contact with the planar face of the horn, and the last sphere in contact with a fixed planar wall. The dynamic displacements of the centers of the consecutive spheres are denoted by $u_{1}, u_{2}, u_{3}, \ldots, u_{n}$, and $u_{0}$ is the input displacement waveform emitted from the boundary (the horn tip). A static applied force $F_{0}$ was modeled by deriving the resulting initial static displacements between the centers of the spheres in the chain. $\delta_{0 l}, \delta_{0}$, and $\delta_{0 r}$ thus denote the mutual approach caused by the static force between the horn and the first sphere, between intermediate spheres, and between the last sphere and the end wall, respectively. The damping force is only considered when the spheres are in contact, and hence a term involving a Heaviside function $H$ on the relative displacement of spheres is incorporated into the equations, as described by Lydon et al. [19]. The damping coefficient was determined depending on the chain structure and the experimental observations, and can be adjusted; for example, the range of $\lambda=0.2-0.4 \mathrm{~N} \mathrm{~s} \mathrm{~m}^{-1}$ was suitably applied for the different length chains consisting of steel spheres of $1 \mathrm{~mm}$ and the polymer holder with Young's modulus, $E_{r}=2.45-3 \mathrm{GPa}$. This was found to give theoretical predictions that were consistent with the observed experimental results. For the first sphere, positioned next to the horn, the equation is

$$
\begin{aligned}
m \frac{d^{2} u_{1}}{d t^{2}}= & \frac{2 \sqrt{R}}{3}\left[2 \theta_{l}\left(\delta_{0 l}+u_{0}-u_{1}\right)^{3 / 2}-\frac{\theta_{m}}{\sqrt{2}}\left(\delta_{0}+u_{1}-u_{2}\right)^{3 / 2}\right] \\
& +\lambda\left(\frac{d u_{0}}{d t}-\frac{d u_{1}}{d t}\right) H\left(\delta_{0 l}+u_{0}-u_{1}\right) \\
& -\lambda\left(\frac{d u_{1}}{d t}-\frac{d u_{2}}{d t}\right) H\left(\delta_{0}+u_{1}-u_{2}\right) .
\end{aligned}
$$

For the second sphere to the penultimate one, the equivalent equation of motion is

$$
\begin{aligned}
m \frac{d^{2} u_{i}}{d t^{2}}= & \frac{\sqrt{2 R}}{3} \theta_{m}\left[\left(\delta_{0}+u_{i-1}-u_{i}\right)^{3 / 2}-\left(\delta_{0}+u_{i}-u_{i+1}\right)^{3 / 2}\right] \\
& +\lambda\left(\frac{d u_{i-1}}{d t}-\frac{d u_{i}}{d t}\right) H\left(\delta_{0}+u_{i-1}-u_{i}\right) \\
& -\lambda\left(\frac{d u_{i}}{d t}-\frac{d u_{i+1}}{d t}\right) H\left(\delta_{0}+u_{i}-u_{i+1}\right) .
\end{aligned}
$$

Finally, for the last (output) sphere, the relevant equation is

$$
\begin{aligned}
m \frac{d^{2} u_{N}}{d t^{2}}= & \frac{2 \sqrt{R}}{3}\left[\frac{\theta_{m}}{\sqrt{2}}\left(\delta_{0}+u_{N-1}-u_{N}\right)^{3 / 2}-2 \theta_{r}\left(\delta_{0 r}+u_{N}\right)^{3 / 2}\right] \\
& +\lambda\left(\frac{d u_{N-1}}{d t}-\frac{d u_{N}}{d t}\right) H\left(\delta_{0}+u_{N-1}-u_{N}\right) \\
& -\lambda \frac{d u_{N}}{d t} H\left(\delta_{0 r}+u_{N}\right) .
\end{aligned}
$$

Here, $\theta_{l}$ and $\theta_{r}$ are the effective Young's moduli associated with contact interactions. The material properties that deter- 
mine the values of some quantities are given by the following equations:

$\frac{1}{\theta_{l}}=\frac{1-v_{l}^{2}}{E_{l}}+\frac{1-v_{s}^{2}}{E_{s}}, \quad \theta_{m}=\frac{E_{s}}{1-v_{s}^{2}}, \quad \frac{1}{\theta_{r}}=\frac{1-v_{r}^{2}}{E_{r}}+\frac{1-v_{s}^{2}}{E_{s}}$,

where $E_{l}$ and $v_{l}$ are the Young's modulus and Poisson ratio of the horn, $E_{r}$ and $v_{r}$ are those of the far end wall, and $E_{s}$ and $v_{s}$ those of the spheres themselves. The values of $\delta_{0 l}, \delta_{0}$, and $\delta_{0 r}$ are given by

$$
\begin{aligned}
& \delta_{0 l}=\left(\frac{3 F_{0}}{4 \sqrt{R} \theta_{l}}\right)^{2 / 3}, \quad \delta_{0}=\left(\frac{3 F_{0}}{\sqrt{2 R} \theta_{m}}\right)^{2 / 3}, \\
& \delta_{0 r}=\left(\frac{3 F_{0}}{4 \sqrt{R} \theta_{r}}\right)^{2 / 3} .
\end{aligned}
$$

Molecular dynamics simulation is an effective approach for solving discrete lattice problems, and hence a Velocity Verlet algorithm [21] was developed to solve the equations of motion numerically. In reality, spheres can lose contact with each other and with the end walls. If this is the case for a particular contact along the chain, there is no force between the two bodies, and the relevant equations would output nonreal complex numbers. However, a condition can be set for Eq. (2), namely, that the contact force is zero if contact is lost, so that the numerical calculation can continue to model the oscillations of individual particles. The relevant material parameters are $E_{l}=E_{s}=$ $201 \mathrm{GPa}$ (steel), and $v_{l}=v_{s}=0.3 ; E_{r}=2.45 \mathrm{GPa}$ and $v_{r}=$ 0.35 (acrylic polymer); the density of steel spheres is $\rho_{s}=$ $7,833 \mathrm{~kg} \mathrm{~m}^{-3}$. The damping coefficient $\lambda=0.32 \mathrm{~N} \mathrm{~s} \mathrm{~m}^{-1}$ was taken to simulate the three different length chains which are presented in the above experimental results.

\section{B. Comparisons between simulations and experiments}

Numerical calculations corresponding to the experiment for the six-sphere chain (Figs. 2 and 3) are presented in Figs. 5-7. For a small amplitude input, a weakly nonlinear behavior was observed experimentally, as was shown earlier in Figs. 2(a) and 3(a). Using zero precompression, the predicted output from the chain is shown in Fig. 5. As might be expected, the predictions differ from those seen experimentally in that the time waveform [Fig. 5(a)] contains some irregular low frequency features, as illustrated by the frequency spectrum of Fig. 5(b); this is because a small initial compression cannot be avoided in the experimental setup. If a very small initial compression force of $0.012 \mathrm{~N}$ is used in the simulations, the resulting predictions are shown in Figs. 6(a) and 7(a). Now the theoretical predictions are more in agreement with the experimental results; the dominant frequency within the output signal is that of the input, with a small amount of harmonics generated due to nonlinearity. This can be attributed to the experimental setup in which a small initial compression is needed to push the spheres together. Accordingly, in our simulation system, very small precompression forces of the order of $0.01 \mathrm{~N}$ are allowed to model the experimental results for different length chains.

Theoretical predictions for intermediate input amplitudes are shown in Figs. 6(b) and 7(b). The predicted output signals match the nonlinear features shown in Figs. 2(b) and 3(b) which were obtained experimentally; i.e., the results represent a kind of nonlinear transitional signal between the weakly nonlinear signals and the strong solitary wave impulses. The time waveform shows some pulselike features, and the spectrum contains some subharmonic content at regularly spaced frequency intervals. At the highest input amplitudes, Figs. 6(c) and 7(c), strongly nonlinear solitary wave impulses are predicted, using the same input conditions as those used to obtain the experimental results of Figs. 2(c) and 3(c). Good agreement is seen.
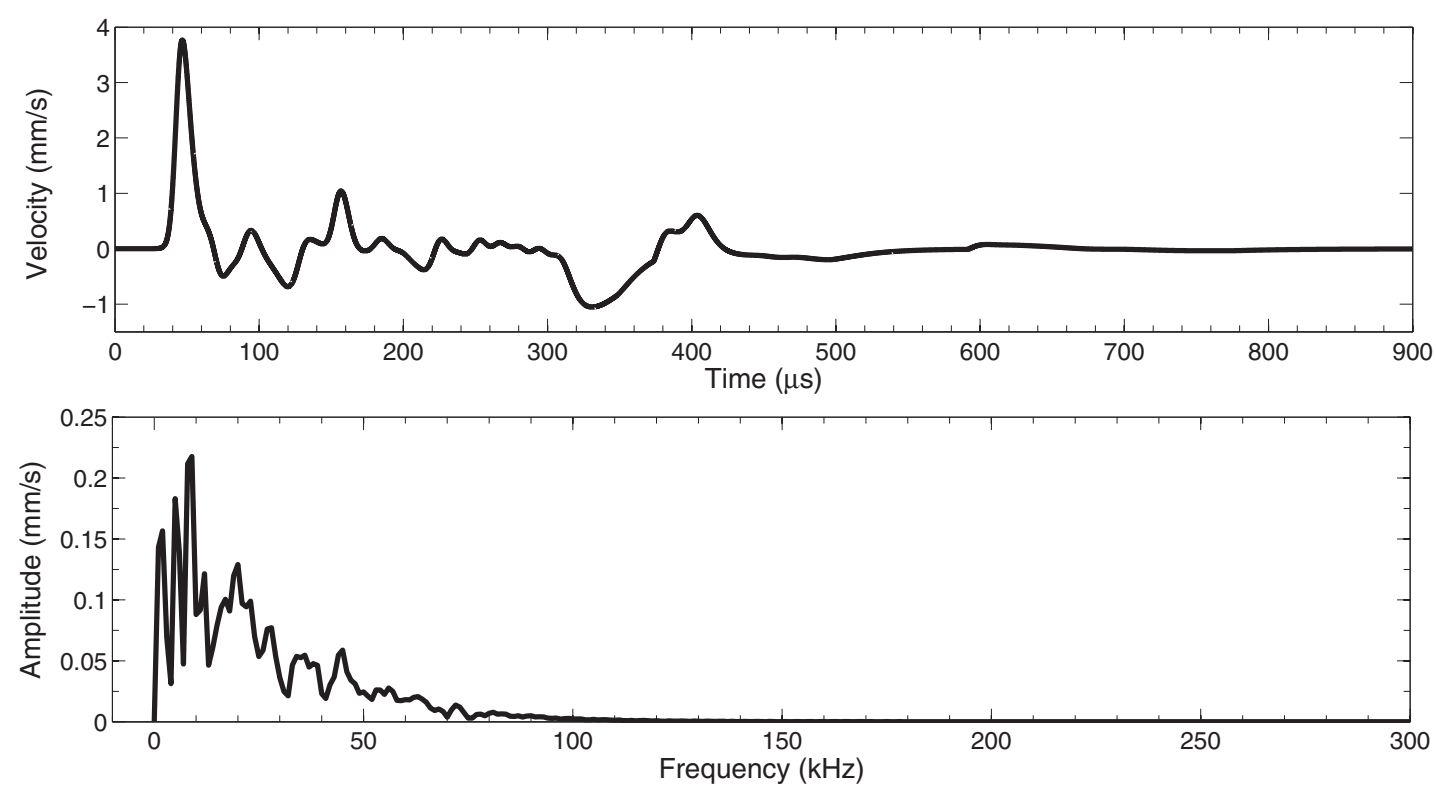

FIG. 5. Numerical results of the output signals of six-sphere chain with a low amplitude input and zero precompression force applied. (a) Time waveform; (b) frequency spectrum. 


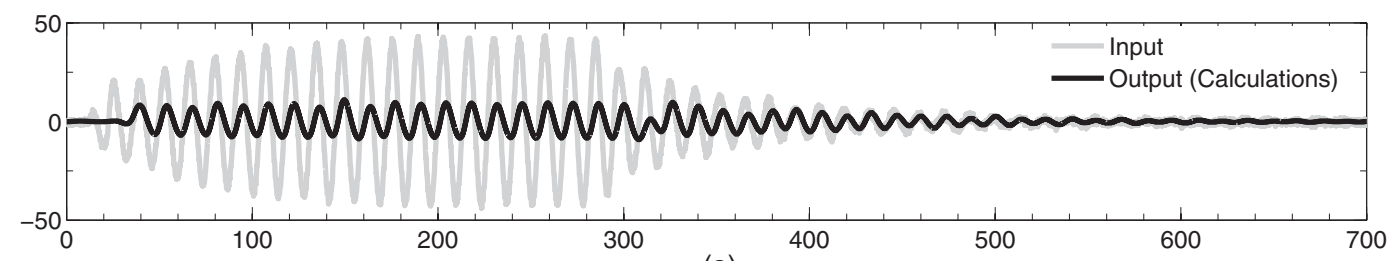

(a)

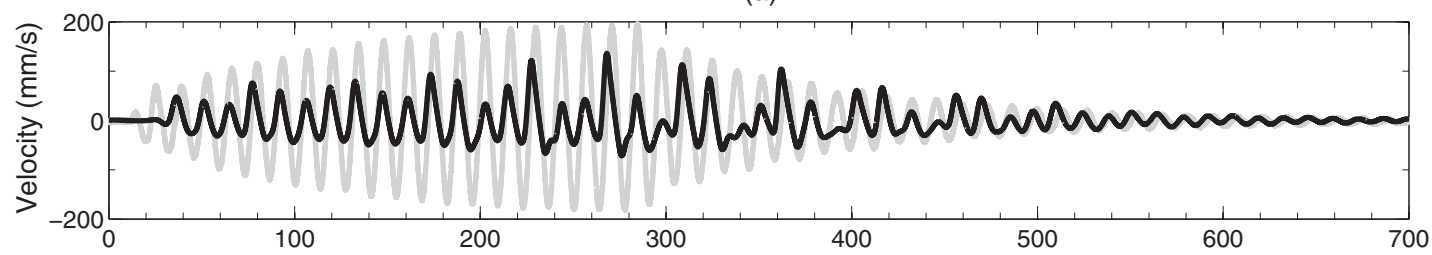

(b)

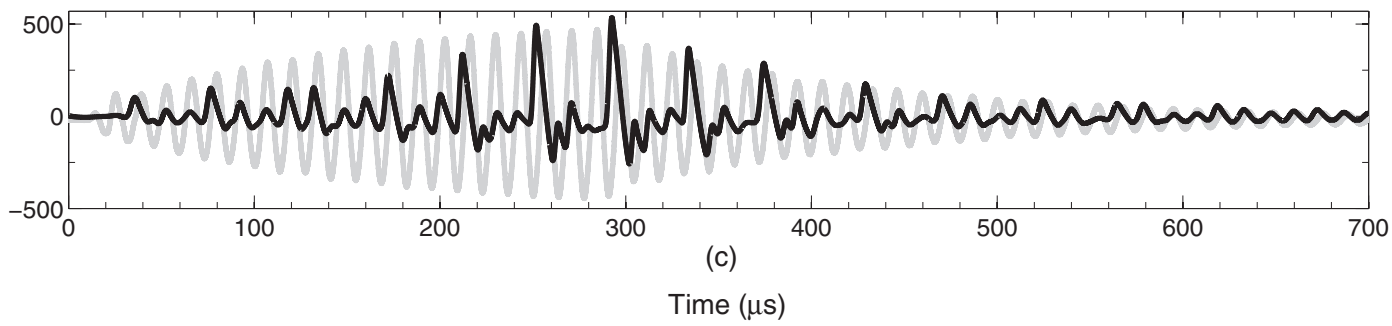

FIG. 6. Predicted output waveforms corresponding to the experimental conditions of Fig. 2.

Numerical calculations for chains of three and ten spheres under the same high amplitude input as the experiments shown in Fig. 4 are presented in Fig. 8. It can be seen that the separation between successive peaks in the frequency spectrum is $1 / 2,1 / 3$, and $1 / 4$ that of the input frequency for chain lengths of three, six, and ten spheres, respectively, as observed experimentally.

\section{Theoretical analysis of solitary wave formation}

Having observed the formation of periodic impulses from a sinusoidal input, it is interesting to understand the propagation characteristics within the chain. Information can be obtained by studying the motion of various spheres within a chain of ten steel spheres of $1 \mathrm{~mm}$ diameter, for the first, fourth, seventh, and tenth (output) sphere. The predictions for the time-domain

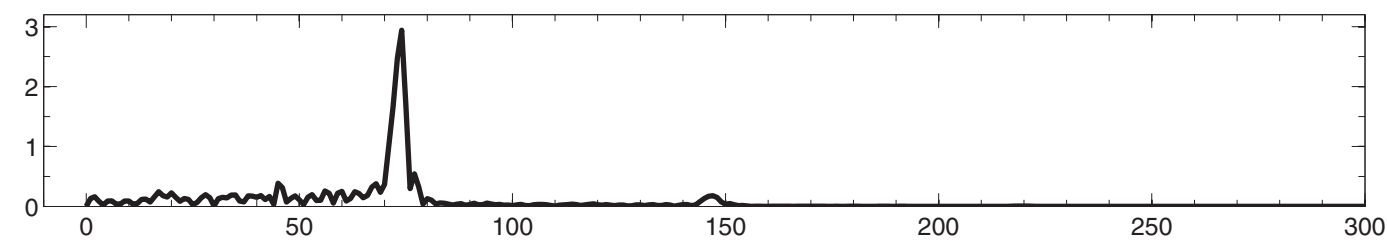

(a)

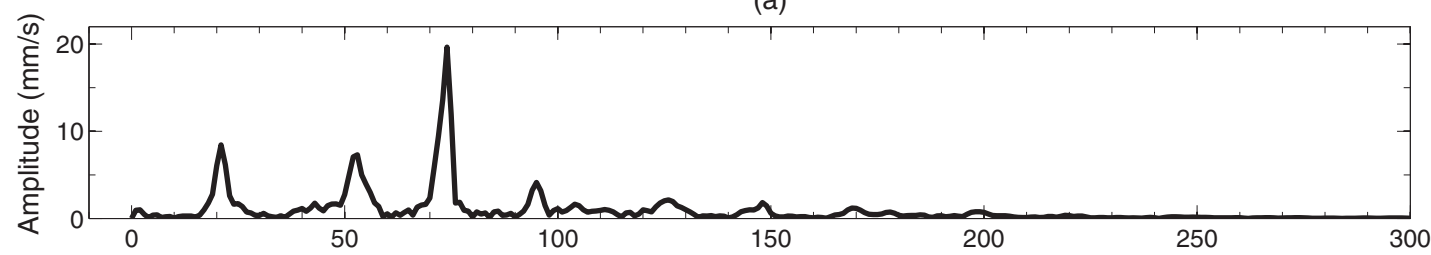

(b)

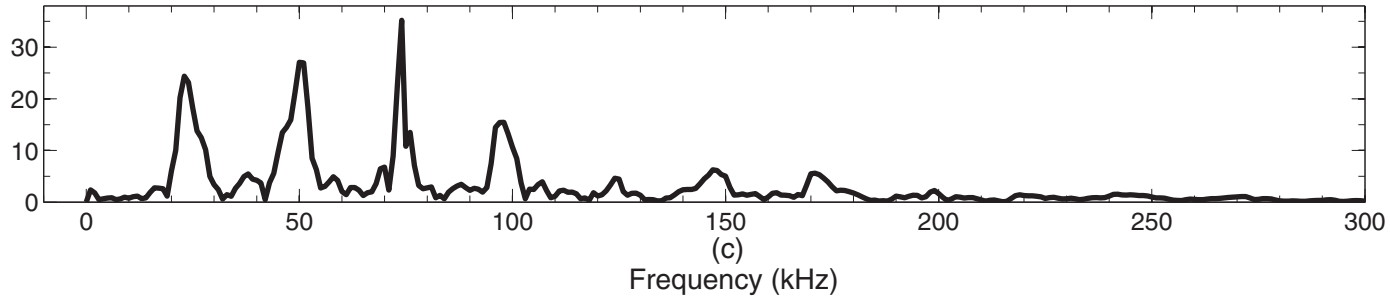

FIG. 7. Frequency spectra obtained from the output signals of Fig. 6. 

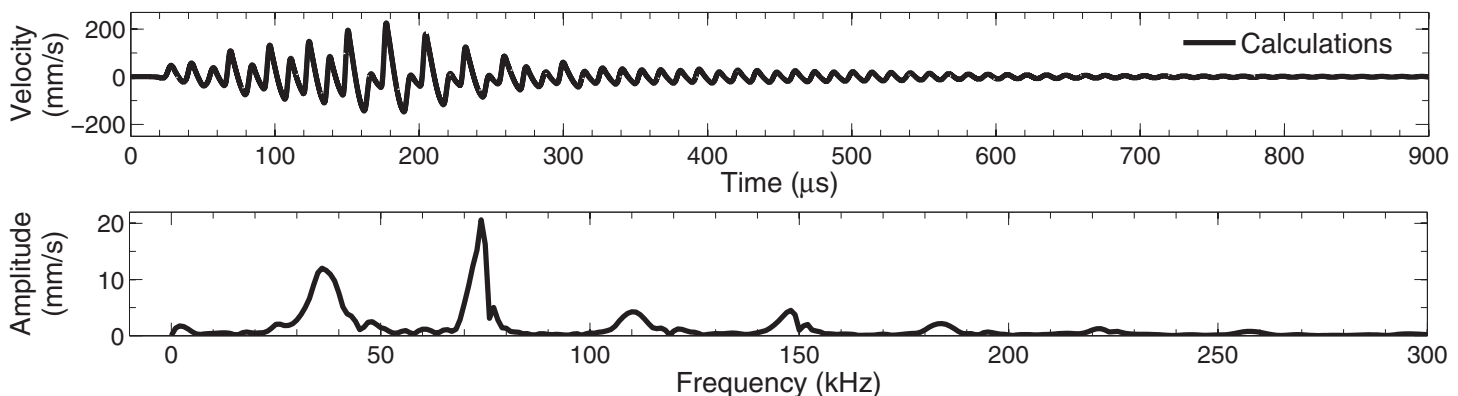

(a)
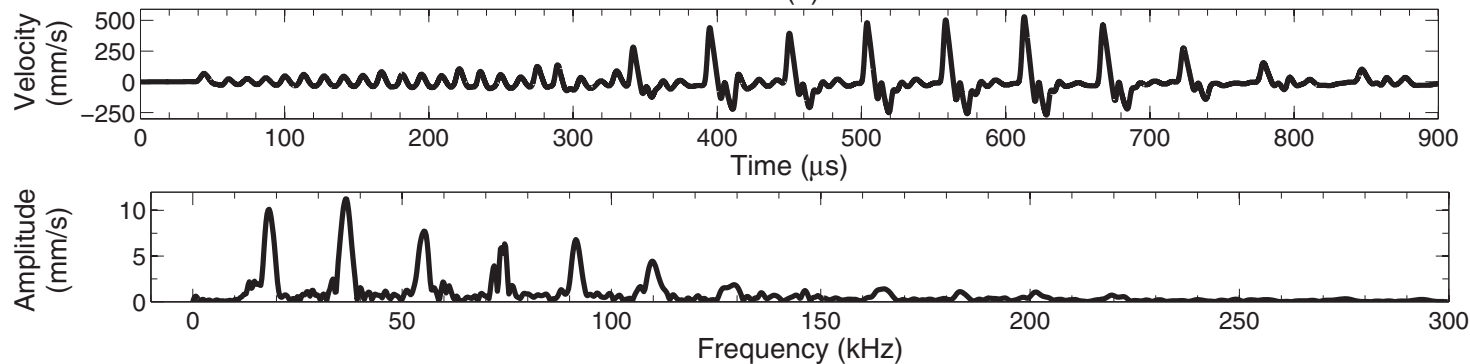

(b)

FIG. 8. Numerical calculations corresponding to the experiments of Fig. 4, for chain lengths of (a) three spheres and (b) ten spheres.

particle velocity waveforms are shown in Fig. 9, the frequency spectra derived from them in Fig. 10, and the corresponding displacement waveforms in Fig. 11.

Consider first the first sphere in the chain. The time waveform of Fig. 9(a) indicates that strong solitary wave impulses build up with time, with a distinct periodicity; the spectrum of Fig. 10(a) indicates the presence of harmonics content, extending beyond $300 \mathrm{kHz}$ in frequency. There are regions of relatively low activity where the particle velocity is close to zero. The same period of solitary wave impulses in the waveforms of other spheres is also observed, which presents the feature of NNM in the discrete dynamics system. Predictions for the fourth sphere show that there is a slight delay for the solitary wave impulses [Fig. 9(b)] compared to the corresponding impulses observed in the first sphere [Fig. 9(a)] and there is some loss of high frequencies [Fig. 10(b)].

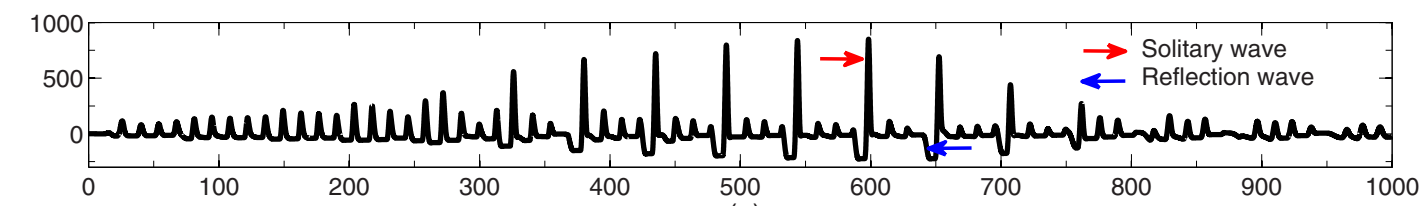

(a)

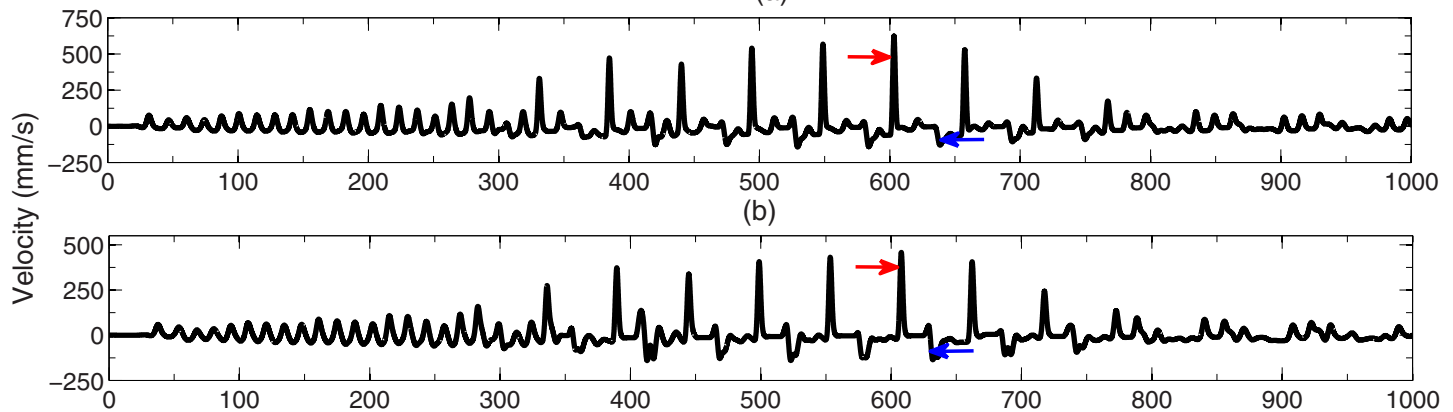

(c)

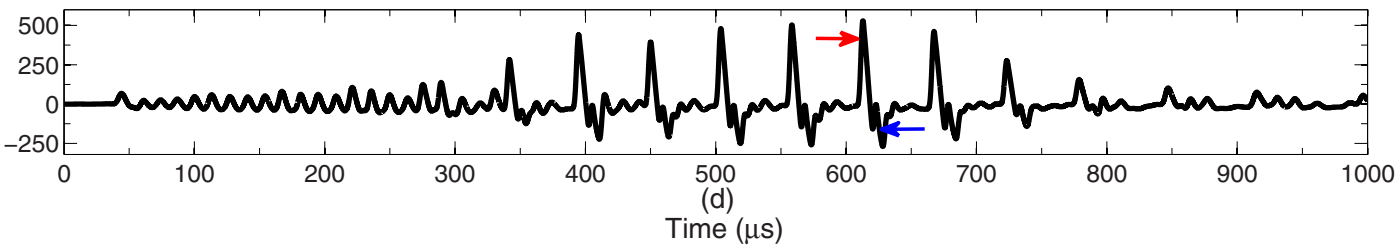

FIG. 9. Predicted waveforms of individual particles within a ten-sphere chain for (a) the first sphere, (b) the fourth sphere, (c) the seventh sphere, and (d) the tenth (last) sphere. The red arrows indicate wave propagation away from the input, and the blue arrows show the signal reflected from the end wall. 


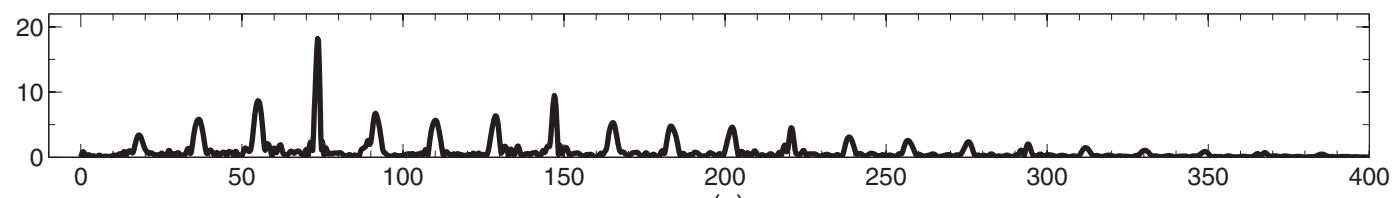

(a)

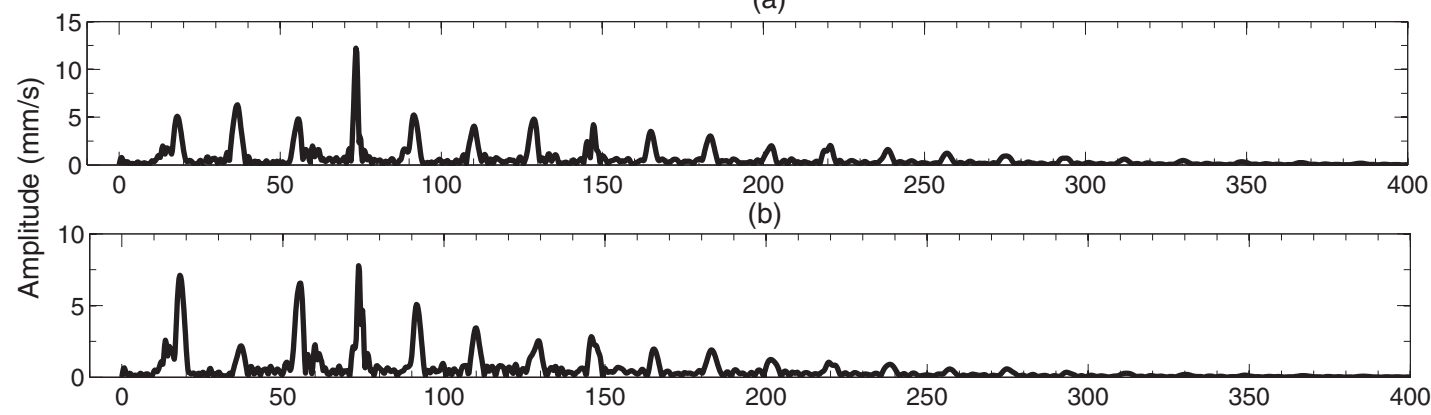

(c)

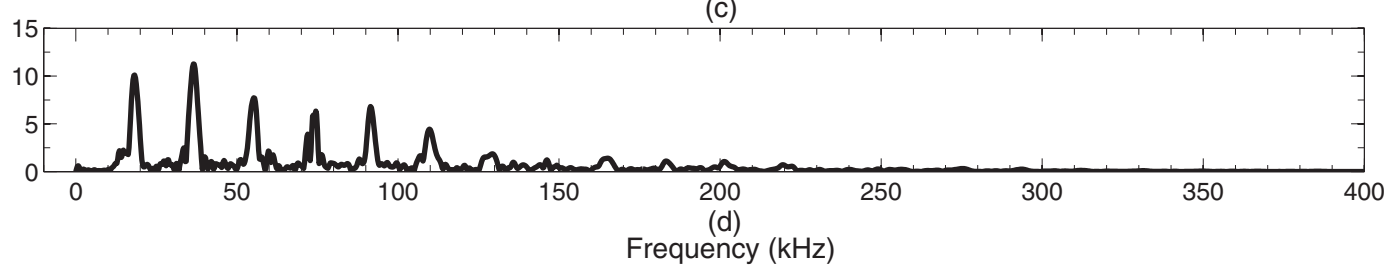

FIG. 10. Calculated spectra of the velocity waveforms of individual particles within a chain of ten spheres for (a) the first sphere; (b) the fourth sphere; (c) the seventh sphere; (d) the last sphere.

The trend continues into the seventh and last spheres. The output [Figs. 9(d) and 10(d)] thus has wider time-domain impulses than those seen in spheres closer to the horn, with a corresponding reduction in frequency content above $150 \mathrm{kHz}$. This is consistent with the use of a dissipation term in the theory, which would selectively attenuate high frequencies.
Note that the output at the tenth sphere has a tendency towards becoming bipolar. This is because of a slightly delayed reflection from the end wall, which is inverted due to a phase change of $\pi$ radians. The reflected wave travels back along the chain. This effect is illustrated by the colored arrows on the time-domain waveforms in Fig. 9 (red for propagation away

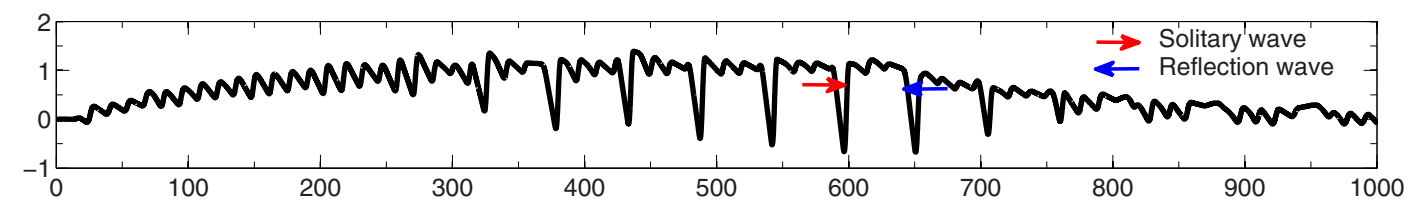

(a)

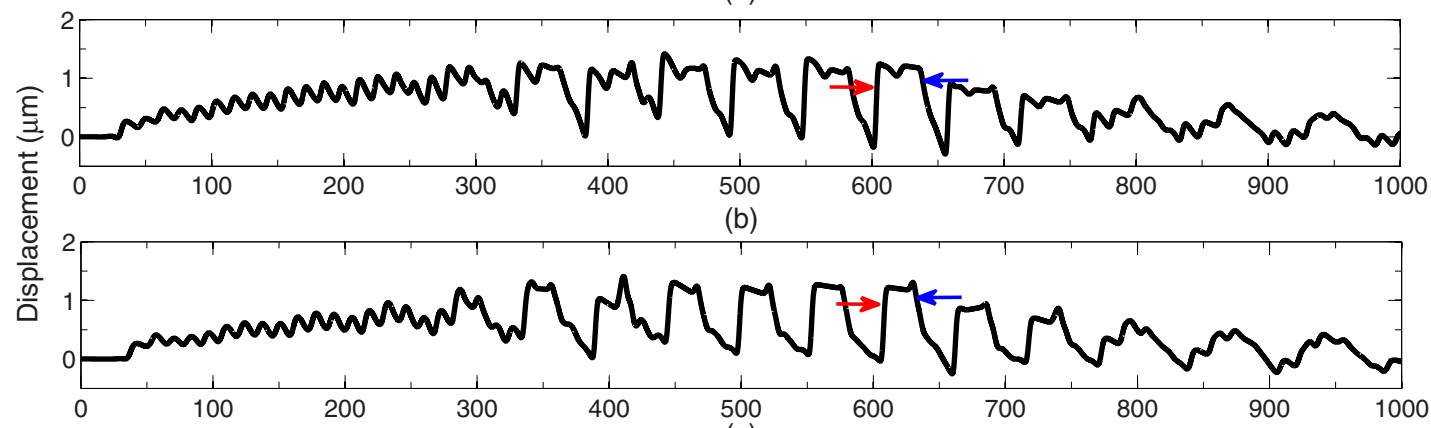

(c)

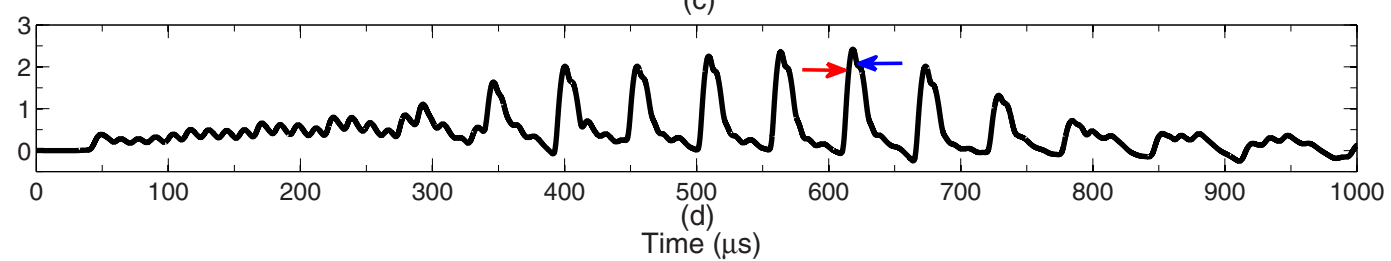

FIG. 11. Displacement of the centers of individual particles within a chain of ten spheres by calculations for (a) the first sphere; (b) the fourth sphere; (c) the seventh sphere; (d) the last sphere. 
from the input, and blue for the reflected signal), and gives the time waveform its characteristic features. In addition, as shown in Fig. 11, the displacements of the centers of the individual spheres are also in the form of periodic waves, but are basically a sequence of positive monopolar signals, which implies that the spheres are in a compressed state due to the transmission of the solitary wave and the reflection wave can make the chain return to its original position.

The above phenomena are consistent with an in-phase NNM of the chain, within which the spheres are allowed to separate. The nonsynchronicity of this in-phase NNM is caused by particle separation, which can lead to "silent" regions in the particle dynamics, whereby one or more particles become motionless for a finite period of time at an offset position from the zero equilibrium [18]. This feature can be observed in Figs. 9 and 11. Thus, the solitary wave impulses can be thought of as an in-phase NNM of the traveling wave in the chain.

Note that the frequency-energy plane of a system containing between two and seven beads was studied theoretically in [18], where it was observed that the in-phase NNM makes a transition toward lower frequencies as the number of beads increased. They also inferred that the in-phase NNM tends towards the zero-frequency axis as the number of beads tends to infinity. The experimental and simulation results correspond well with this conclusion.

\section{COMPARISON TO EXISTING ANALYTICAL MODELS FOR SOLITARY WAVES IN GRANULAR CHAINS}

The aim of this part of the work was to study the differences between our findings and the characteristics of solitary wave propagation reported by previous workers in this area [1-9]. In the present study, periodic impulsive signals are present as an in-phase NNM in both experiments and numerical calculations, and it is interesting to establish the existence of solitary waves in such a dispersive and dissipative granular system. The conditions to generate the periodic nature of our signals will be discussed later.

Consider first the approach of existing analytical models for a chain of spheres of infinite length, when it is "weakly compressed," i.e., when $\left|u_{i}-u_{i-1}\right| \gg \delta_{0}[1,4]$. A long-wavelength approximation is made and in the derivation of the wave equation, $u_{i}(t)$ in the dynamic equations may be written as $u(x, t)$; the displacements $u_{i-1}$ and $u_{i+1}$ are then expanded in a power series to the fourth order. Accordingly, the wave equation is [4]

$$
\begin{aligned}
u_{t t}= & c^{2}\left[\frac{3}{2}\left(-u_{x}\right)^{1 / 2} u_{x x}+\frac{a^{2}}{8}\left(-u_{x}\right)^{1 / 2} u_{x x x x}\right. \\
& \left.-\frac{a^{2}}{8} \frac{u_{x x} u_{x x x}}{\left(-u_{x}\right)^{1 / 2}}-\frac{a^{2}}{64} \frac{\left(u_{x x}\right)^{3}}{\left(-u_{x}\right)^{3 / 2}}\right]
\end{aligned}
$$

Where $-u_{x}>0, \quad c^{2}=\frac{2 E}{\pi \rho_{s}\left(1-v^{2}\right)}, \quad a=2 R$.

Friction and viscoelastic dissipation are not taken into account in the original discrete dynamic equations from which Eq. (5) was derived. In order to obtain the solution of a traveling wave with velocity $V$, a transformation of the form $u(x-V t)$ with a new variable strain $\xi=-u_{x}$ changes Eq. (5) into

$$
\frac{V^{2}}{c^{2}} \xi_{x}=\frac{3}{2} \xi^{1 / 2} \xi_{x}+\frac{a^{2}}{8} \frac{\left(\xi \xi_{x x}\right)_{x}}{\xi^{1 / 2}}-\frac{a^{2}}{64} \frac{\xi_{x}^{3}}{\xi^{3 / 2}} .
$$

While the initial compression strain of the chain $\xi_{0}$ is extremely small, the strain and the particle velocity $v$ of the solitary waves can be solved from Eq. (6), and the profile is taken as one monopolar amplitude excursion of a periodic solution given by Eqs. (7a) and (7b):

$$
\begin{aligned}
& \xi=\left(\frac{5 V_{s}^{2}}{4 c^{2}}\right)^{2} \cos ^{4}\left(\frac{\sqrt{10}}{5 a} x\right), \\
& v=V_{s}\left(\frac{5 V_{s}^{2}}{4 c^{2}}\right)^{2} \cos ^{4}\left(\frac{\sqrt{10}}{5 a} x\right) .
\end{aligned}
$$

The phase speed $V_{s}$ of a solitary wave has a nonlinear dependence on the maximum strain or the maximum particle velocity $v_{m}$ in the wave:

$$
V_{s}=\frac{2}{\sqrt{5}} c \xi_{m}^{1 / 4}=\left(\frac{16}{25}\right)^{1 / 5} c^{4 / 5} v_{m}^{1 / 5} .
$$

The characteristic spatial size of a soliton $L_{s}$ is determined by the period of the solution described by Eq. (7), which equals

$$
L_{s}=\left(\frac{5 a}{\sqrt{10}}\right) \pi \approx 5 a .
$$

Consider now chain lengths of three, six, and ten spheres to study the characteristics predicted by the above analytical solution of solitary wave under our experimental conditions. The phase velocity of the solitary wave was calculated from the maximum particle velocity using Eq. (8), from which the particle velocity profile of the solitary wave can be calculated using Eq. (7b). Here we assume that the maximum velocity in Eq. (8) is the same as the maximum value of the velocity impulses of the first sphere in the numerical simulations; this is done because of energy dissipation at later spheres in the chain. A particular impulse with the maximum amplitude in the sequence can be chosen (for example, the impulse indicated by the red arrow in the ten-sphere chain shown in Fig. 9) for the three chains, respectively. The phase speed $V_{s}$ solved by Eq. (8) for the particular impulses are 568, 674, and $706 \mathrm{~ms}^{-1}$ for chains containing three, six, and ten spheres, respectively. The profiles of solitary waves traveling from the first sphere to the last sphere in the three different length chains as predicted by the long-wavelength approximation solution described by Eqs. (7) and (8) are shown in Figs. 12(a), 13(a), and 14(a), respectively.

In the numerical calculations demonstrated in Fig. 9, the particle velocity waveform was calculated for each sphere in a particular chain as a function of time. Using our discrete model, it can now be determined whether each of the impulses in each time waveform has similar characteristics to those predicted above for a solitary wave. Assume that the outwardly propagating wave is represented by the positive (monopolar) part of each impulse in a particular waveform, as discussed above. A particular impulse (the same as the above) and its form for successive spheres in the chain can again be plotted. The results for the three different chain lengths are shown in Figs. 12(b), 13(b), and 14(b), respectively, to compare to 


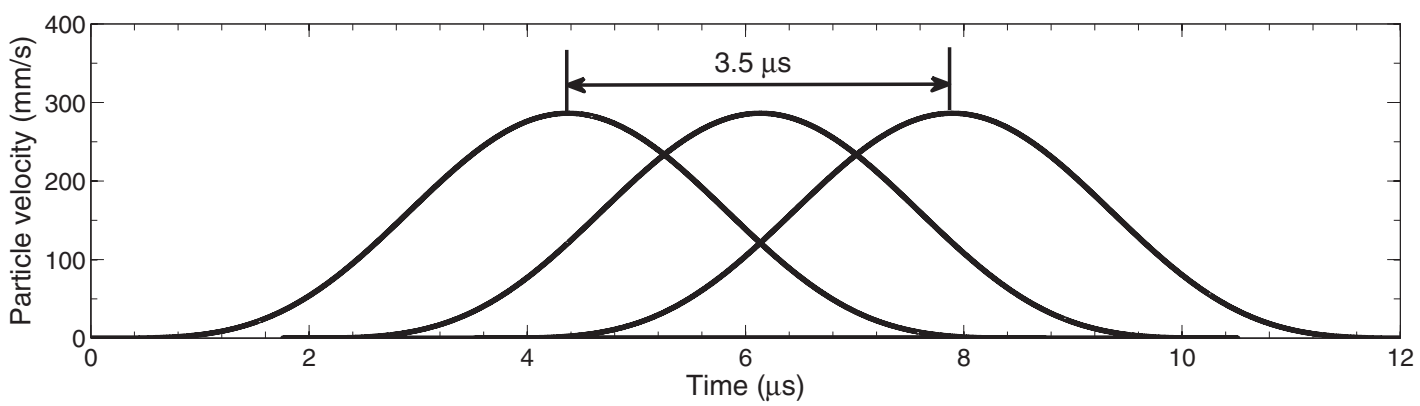

(a)

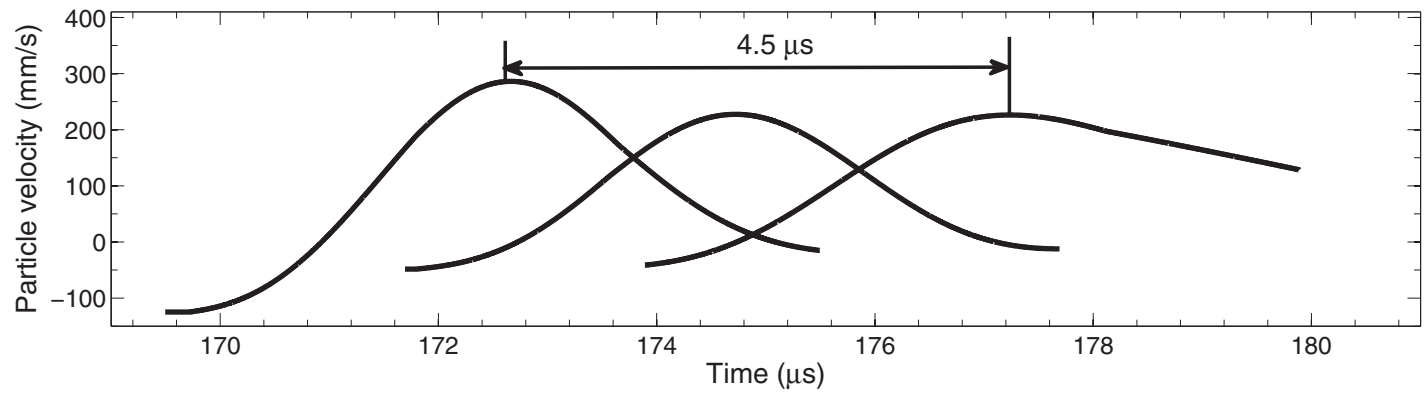

(b)

FIG. 12. Partial particle velocity waveforms for a specific peak in the predicted waveform for each adjacent sphere in a chain of three 1-mm-diameter steel spheres. (a) Solitary wave profiles calculated using the long-wavelength approximation of Eqs. (7) and (8); (b) numerical calculations of the wave formed by the maximum velocity profiles of three adjacent spheres for a particular impulse using Eqs. (2)-(4).

predictions of the analytical model, and the resultant phase velocities are 444,588 , and $621 \mathrm{~ms}^{-1}$, respectively, for the chains with three, six, and ten spheres. For a longer chain, the results are closer to the analytical solution. Note that dissipation has caused the peak amplitudes to decrease as the chosen impulse moves further along the chain.

The phase velocities of three, five, and six individual consecutive impulses in the middle of the three different chains are also investigated, respectively, in both solutions of the numerical calculations and the long-wavelength approximation, and the results are shown in Fig. 15. The mean phase velocity in both solutions is slightly smaller than that of the impulse chosen above, whose particle velocity amplitude is greater than the other impulses in the sequence, and this result can be attributed to the output characteristics of the horn. As seen in Fig. 2, the particle velocity of the horn measured by the laser

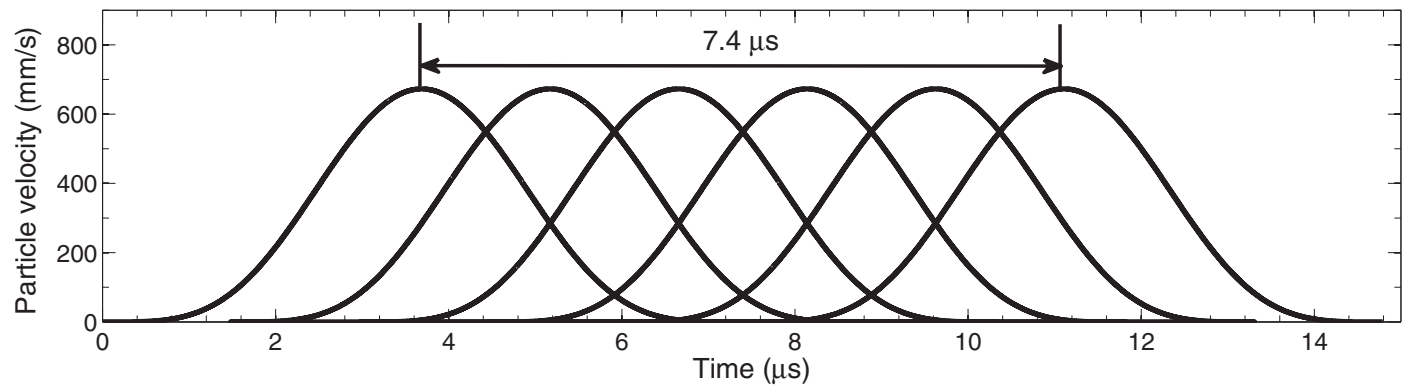

(a)

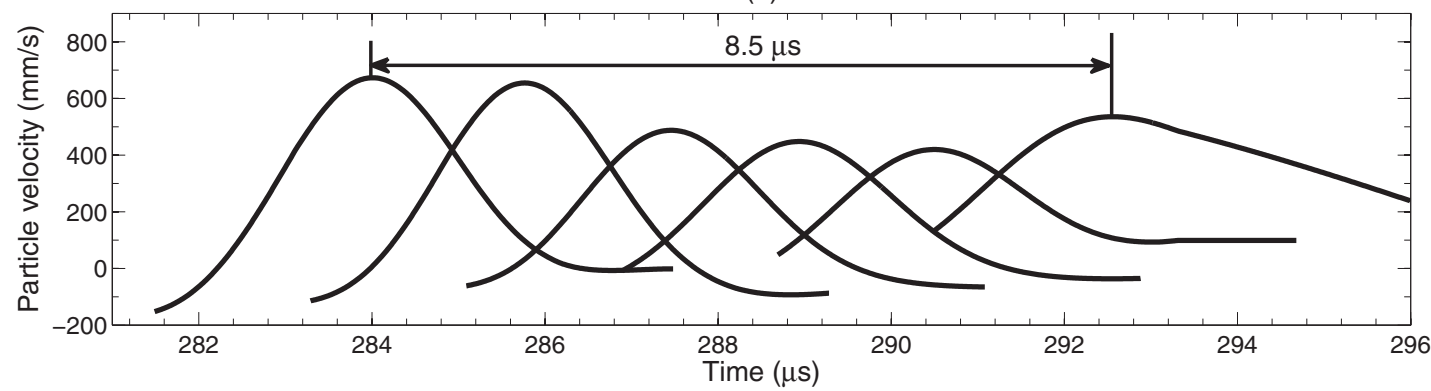

(b)

FIG. 13. As Fig. 12, but for six adjacent spheres in a six-particle chain. 


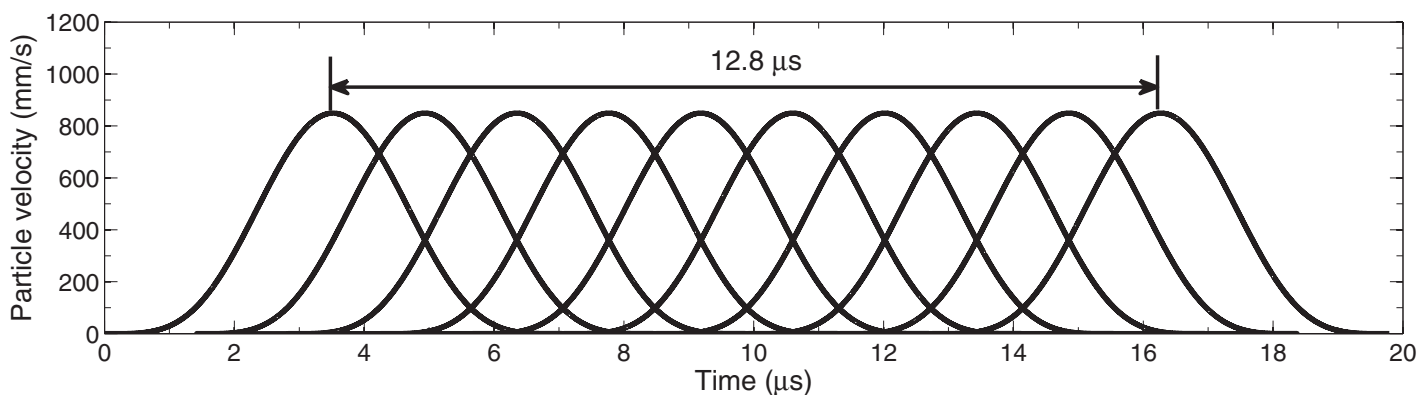

(a)

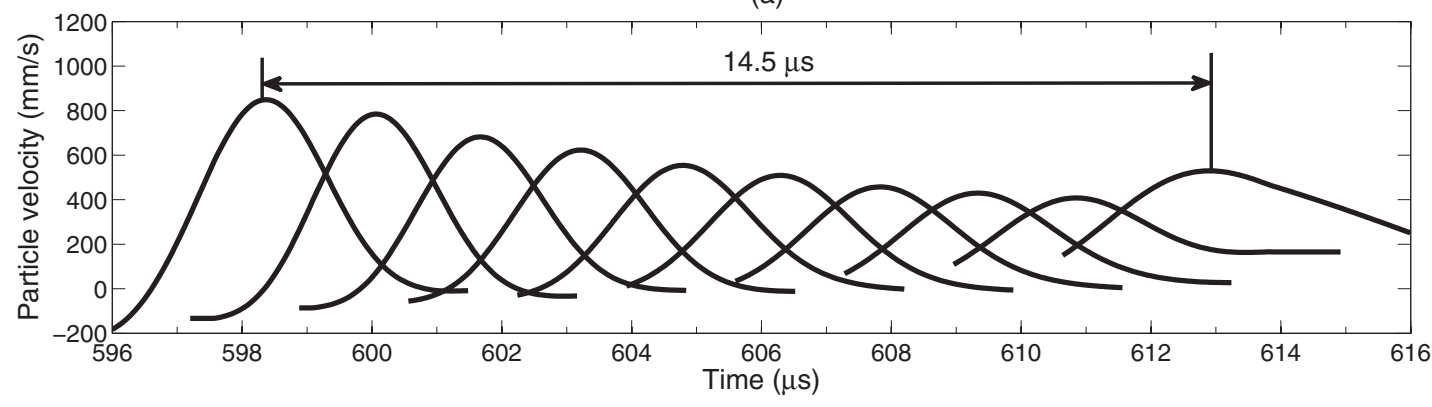

(b)

FIG. 14. As Fig. 12, but for ten adjacent spheres in a ten-particle chain.

vibrometry changes amplitude with time. When the amplitude of the input signals attains a certain value, a solitary wave can be produced; however, as the phase velocity of a solitary wave is also related to the amplitude of the input pulse, each solitary wave impulse is likely to have a slightly different phase velocity. In general, the phase velocities estimated by the numerical calculations are smaller than those predicted by the analytical solution, due to the dissipation modeled by the damping effect in the numerical calculations.

In addition, using the mean phase velocities obtained from both solutions, we choose the three, five, and six individual consecutive impulses exhibited in Figs. 2(c) and 4 and calculate the characteristic widths of the positive-going impulses of the experimental signals for the three chain lengths, respectively. The results are shown in terms of the equivalent number of sphere diameters in Fig. 16. The width predicted from Eq. (9) of the analytical model is close to five spheres. Experimentally, the width varies as the impulses build up with time, and also vary between the three chain lengths. However, the average values obtained from the mean phase velocity solved in the method of the long-wave approximation are much closer to that expected analytically, especially for the three-sphere chain and the ten-sphere chain. The characteristic widths obtained using the mean phase velocity solved by numerical calculations are

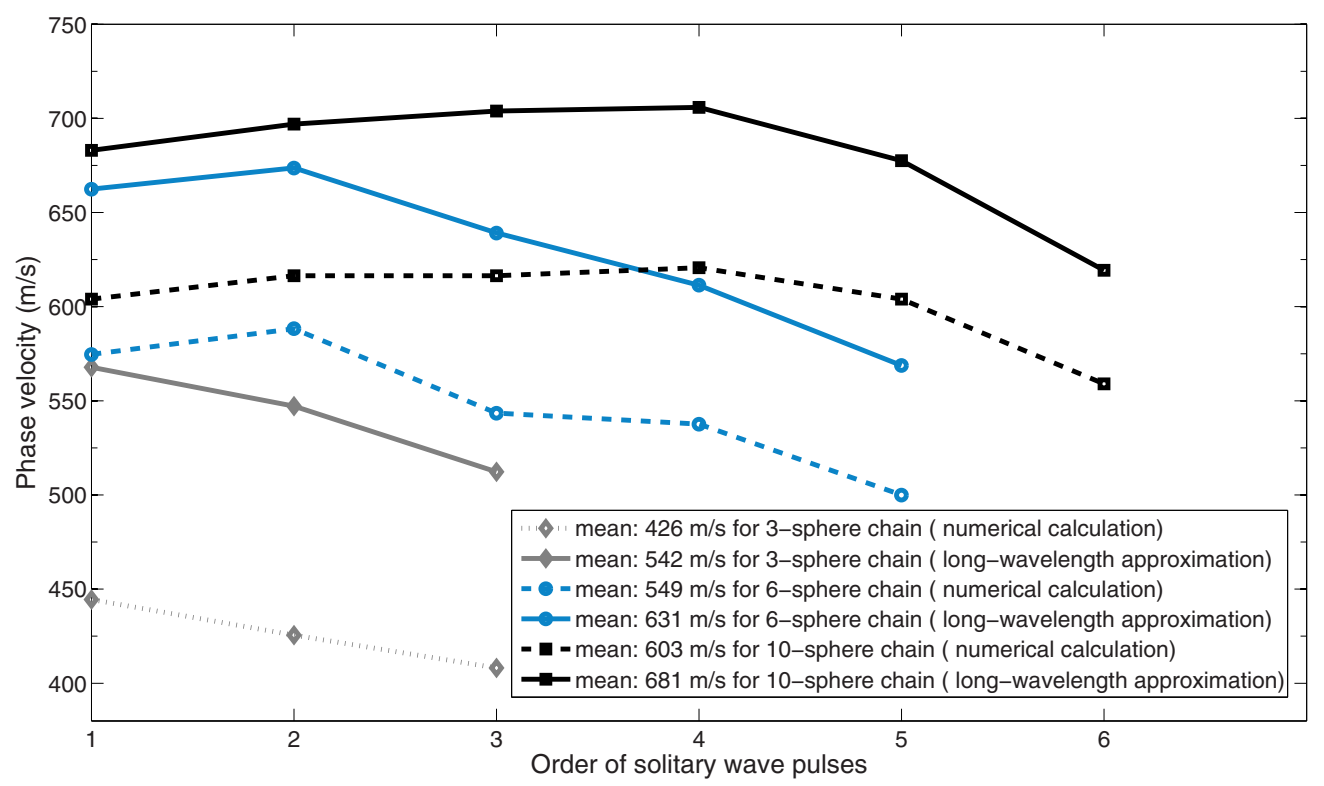

FIG. 15. Phase velocity of three impulses in the three-sphere chain, five impulses in the six-sphere chain, and six impulses in the ten-sphere chain, respectively, which are solved by both solutions of the numerical calculation and the long-wavelength approximation. 


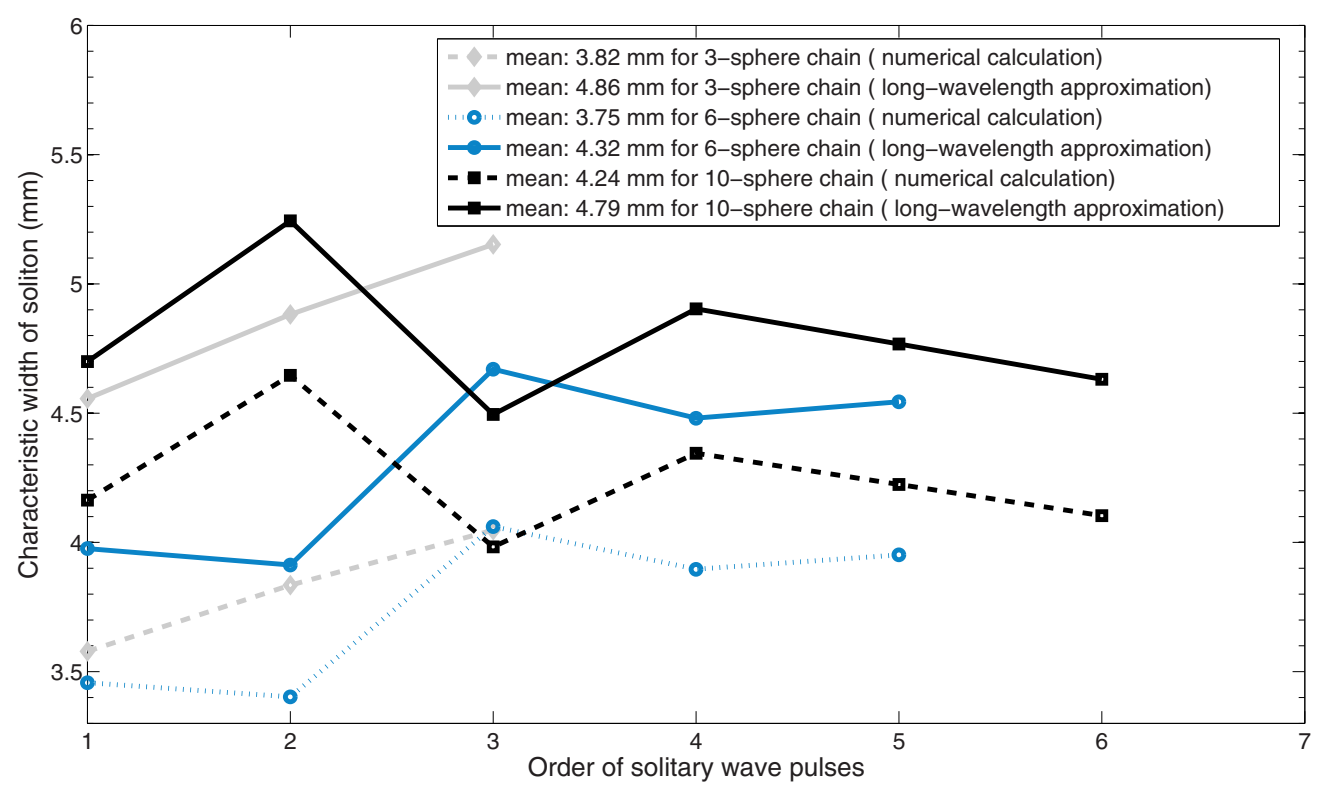

FIG. 16. The estimated characteristic sizes of three impulses in the three-sphere chain, five impulses in the six-sphere chain, and six impulses in the ten-sphere chain, which are chosen from the experimental results in Figs. 2(c) and 4, respectively.

smaller than the results of the long-wavelength approximation, but not too dissimilar to the analytical solution. This result is also regarded as the effect of the dissipation modeled in Eq. (2). The solitary wave in Nesterenko's derivation $[1,4]$ corresponds to the maximum potential energy within a mechanical analogy, and in a special case, a single monopolar part of a periodic solution may represent solitary solutions for infinitesimally small initial prestrains [4]. Correspondingly, in the extreme case of a single impulse, solved in our models for a certain chain structure using Eq. (2) [as shown in Figs. 12(b), 13(b), and 14(b)], it also exhibits the feature of a solitary wave. For a system without dissipation, the traveling wave may always propagate back and forward through a finite-length chain under the law of conservation of energy. In a general experimental system, the dissipation due to friction will result in the attenuation of the output signals in the chain when the input signal stops, so in the previous experimental studies of the solitary waves [1-9], no periodic solitary wave impulses have been observed. It is an interesting question as to what input conditions are necessary for the periodic solitary wave impulses to be generated.

As shown in the output signals in Fig. 2(c), the period of the impulses depends on the characteristic time of the solitary wave and the characteristic time of the reflection wave as well as the silent time, i.e., the transmission time of the traveling wave in the chain. For any other specific sphere in the chain the recurrence of the solitary wave appears after a silent period and a reflection wave profile, as shown in Fig. 9. When the solitary wave propagates to the end of the chain, it reflects and a new reflection wave is produced due to the action of the fixed end of the chain on the last sphere, and the reflection wave starts to propagate back along the chain towards the source. As the reflected wave arrives, the spheres gradually return to their original positions. As seen in Fig. 9, the amplitude of the signal reflected from the end wall is much smaller than that of the original wave from the source; hence, this reflected wave needs more time to travel a certain distance along the chain. Eventually, the reflected wave reaches the first sphere in the chain, and spheres throughout the whole chain have returned to a position close to their original starting position. Energy input from the source then promotes the generation of a new solitary wave. This solitary wave complies with the same transfer mode as the first solitary wave. This procedure is repeated, and the number of the impulses generated depends directly on the frequency of the input signal relative to the chain properties, and is also related to the time duration of the input signals.

Another interesting result is that the period of the solitary wave impulses in finite-length chains is an integer multiple of the period of the input signal, and forms different subharmonics in different lengths of chain. This can be explained by the need to match outward solitary waves and reflected signals in a resonant system, and to return all spheres to their initial positions by the arrival of the wave reflected back to the source. Accordingly, specific conditions are required to match the period of the input signal to the chain propagation characteristics, to enable solitary wave impulses to form. This is also the reason why the solitary wave impulses favor certain chain lengths, such as the three-, six-, and ten-sphere chains for input at $73 \mathrm{kHz}$. This allows the system to produce the fundamental frequencies of $1 / 2,1 / 3$, and $1 / 4$ of the input frequency, respectively, and hence to produce the characteristic form of the solitary wave impulses in finite-length chains.

\section{v. CONCLUSIONS}

The main discovery in this paper is that a train of solitary wave impulses with a known periodicity can be generated within finite lengths of chain, and that it is possible to predict their characteristics theoretically and understand their behavior. These solitary wave impulses exhibit different characteristics from the traditional solitary waves studied in granular media. Previous experimental studies use impulsive 
excitation, so that the signals generated by the propagation of solitary waves in granular chains are usually nonperiodic. However, we obtained high frequency impulses with a certain periodicity using continuous ultrasonic excitations, and we have shown here that each impulse demonstrates the feature of solitary wave propagation. The properties of the chain structure can be adjusted so as to generate ultrasonic impulses with a high amplitude and known frequency content (including both harmonics and subharmonics of the input frequency), and exhibit great potential in applications such as high intensity focused ultrasound (HIFU), where higher ultrasonic frequencies are required.

We identified the differences between our results and traditional solitary waves. The latter can be explained by a stationary solution of the sonic vacuum wave model. However, this continuum model does not consider the combined effects of dissipation and separation between spheres in a finite-length chain (in fact it assumed a chain of infinite length, where the periodicity of impulses observed in our experiments would not be predicted). The discrete dynamic equations used here not only describe these effects, but allow the addition of a small applied static force to be studied. The numerical results based on our equations are consistent with the experimental results. The phase velocity of a solitary wave propagating along the chain predicted by the numerical solution is smaller than that predicted by existing analytical solutions, and the characteristic size of the soliton is also slightly smaller than that predicted by the analytical model. These differences may be attributed to the damping introduced into the equations.

The paper has studied the motion of individual spheres in a chain, and hence determined why these solitary wave impulses are created from a very narrow bandwidth input. Due to the high amplitude sinusoidal input in weakly compressed chains, separation between both adjacent spheres and between the end spheres and the transducer or fixed end can occur. However, the discontinuous nature of the dynamics within the chain can be solved by numerical calculations. This has allowed us to explain the form of the signals that resulted from the outgoing solitary wave interacting with a much weaker reflected signal. In the dissipative experimental system, the solitary wave reflects back along the chain towards the input, due to the fixed end in a finite-length chain. The continued pulses of ultrasonic excitations will act on the first sphere to generate a new solitary wave after the reflection wave propagates to the first sphere to make the chain return to close to its initial position. This leads to the possibility that the period of the solitary wave impulses is an integral multiple of the period of the input signal, and hence forms different subharmonics within the frequency spectrum in different length chains under some specific conditions. These specific conditions imply that the chain structure needs to match the input ultrasonic signals to generate the solitary wave impulses. The resultant periodic solitary wave impulses can also be regarded as the propagation of a traveling wave in an in-phase NNM, characterized by a series of allowed frequency modes as both harmonics and subharmonics of the input frequency.

In summary, the generation of the solitary wave impulses depends on two factors. The first includes the properties of the chain, in terms of the materials used; the size of the spheres and the length of the chain, etc., determines the dynamics of the granular media. The second includes external factors, such as the amplitude and frequency of the resonant transducer and the time duration of the input signals, which trigger the natural dynamics of chains. It is important that these two factors match for a given chain, in order to achieve the desired effects.

\section{ACKNOWLEDGMENT}

The authors gratefully acknowledge funding from the Engineering and Physical Sciences Research Council (UK) via Grant No. EP/K030159/1. All relevant data are provided in full in the results section of this paper.
[1] V. F. Nesterenko, J. Appl. Mech. Tech. Phys. 24, 733 (1983).

[2] C. Coste, E. Falcon, and S. Fauve, Phys. Rev. E 56, 6104 (1997).

[3] A. Chatterjee, Phys. Rev. E 59, 5912 (1999).

[4] V. F. Nesterenko, Dynamics of Heterogeneous Materials (Springer, New York, 2001).

[5] C. Daraio, V. F. Nesterenko, E. B. Herbold, and S. Jin, Phys. Rev. E 72, 016603 (2005).

[6] S. Job, F. Melo, A. Sokolow, and S. Sen, Phys. Rev. Lett. 94, 178002 (2005).

[7] V. F. Nesterenko, C. Daraio, E. B. Herbold, and S. Jin, Phys. Rev. Lett. 95, 158702 (2005).

[8] E. B. Herbold and V. F. Nesterenko, Appl. Phys. Lett. 90, 261902 (2007).

[9] S. Sen, J. Hong, J. Bang, E. Avalos, and R. Doney, Phys. Rep. 462, 21 (2008).

[10] V. F. Nesterenko and E. B. Herbold, Phys. Procedia 3, 457 (2010).

[11] C. Daraio, V. F. Nesterenko, E. B. Herbold, and S. Jin, Phys. Rev. E 73, 026610 (2006).
[12] A. Spadoni and C. Daraio, Proc. Natl. Acad. Sci. USA 107, 7230 (2010).

[13] C. M. Donahue, P. W. J. Anzel, L. Bonanomi, T. A. Keller, and C. Daraio, Appl. Phys. Lett. 104, 014103 (2014).

[14] R. S. Mackay, Phys. Lett. A 251, 191 (1999).

[15] J. Y. Ji and J. Hong, Phys. Lett. A 260, 60 (1999).

[16] G. Friesecke and J. A. D. Wattis, Commun. Math. Phys. 161, 391 (1994).

[17] Y. Starosvetsky and A. F. Vakakis, Phys. Rev. E 82, 026603 (2010).

[18] K. R. Jayaprakash, Y. Starosvetsky, A. F. Vakakis, M. Peeters, and G. Kerschen, Nonlinear Dyn. 63, 359 (2011).

[19] J. Lydon, K. R. Jayaprakash, D. Ngo, Y. Starosvetsky, A. F. Vakakis, and C. Daraio, Phys. Rev. E 88, 012206 (2013).

[20] D. A. Hutchins, J. Yang, O. Akanji, P. J. Thomas, L. A. J. Davis, S. Freear, S. Harput, N. Saffari, and P. Gelat, Europhys. Lett. 109, 54002 (2015).

[21] N. S. Martys and R. D. Mountain, Phys. Rev. E 59, 3733 (1999). 